\section{Free-standing all-polymer microring resonator optical filter}

\section{G.T. Paloczi, Y. Huang and A. Yariv}

Free-standing all-polymer microring resonator optical filters as prototypical elements in flexible integrated lightwave circuits are demonstrated. The fabrication and measurement methods are discussed. The measured spectrum shows good agreement with theoretical expectations. The crucial 'critical' coupling condition is achieved, resulting in a measurement limited $-27 \mathrm{~dB}$ extinction of the filter output on resonances.

Introduction: Polymeric optical devices have enjoyed steady progress towards the goal of their deployment in commercial optical communications applications. In particular, recently polymeric optical modulators have demonstrated low switching voltage [1] and high modulation bandwidth [2]. These demonstrations are testament to both the remarkable optical nonlinearity of doped polymeric materials as well as the exceptional inherent material bandwidth. Another important property of polymers that differs from that of crystalline materials used in optical devices is the soft and flexible nature of the material. In this Letter, we show that, by peeling a film of devices from the substrate upon which the devices are fabricated, we are able to produce free-standing all-polymer integrated optical devices. To demonstrate the optical quality of these materials we chose to fabricate optical notch filters that are based on waveguide-to-microring resonator coupling. Such devices depend on exquisite control of the coupling region to achieve the requisite field coupling ratio for good optical performance and thus constitute an important test case as canonical elements in integrated optical lightwave circuits.

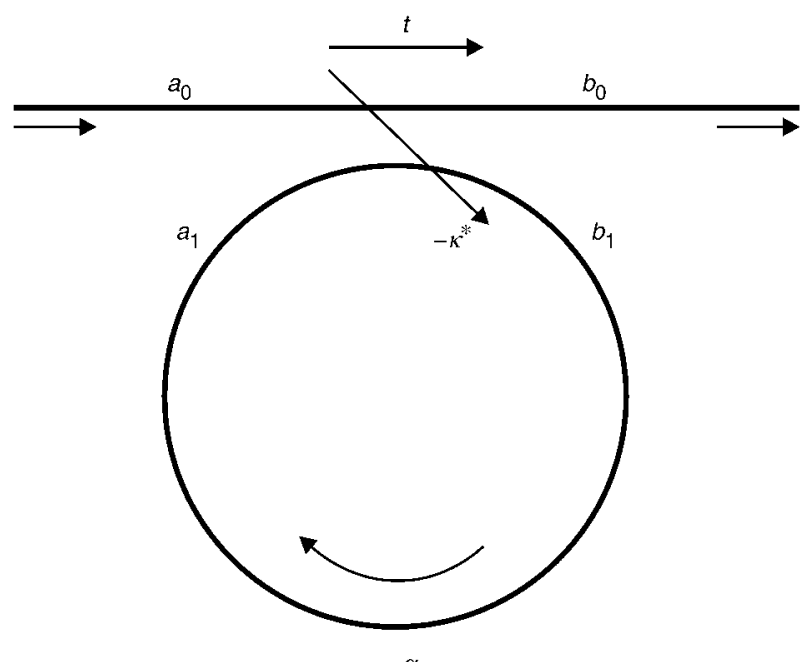

Fig. 1 Prototypical optical filter comprising waveguide side-coupled to microring resonator

Theory: Shown in Fig. 1 is the simplest microring resonator filter with a single side-coupled waveguide acting as both input and output. The matrix representing the coupling of light from the waveguide to the ring is [3]

$$
\left[\begin{array}{l}
b_{0} \\
b_{1}
\end{array}\right]=\left[\begin{array}{cc}
t & \kappa \\
-\kappa^{*} & t^{*}
\end{array}\right]\left[\begin{array}{l}
a_{0} \\
a_{1}
\end{array}\right]
$$

with $\kappa$ denoting the coupling coefficient and $t$ the field amplitude transmission past the coupling region. Assuming the case of lossless coupling, we take $|t|^{2}+|\kappa|^{2}=1$. Referring to Fig. 1, $\alpha$ represents the portion of the field amplitude remaining after one round-trip of length $L$ in the resonator and $\beta \cdot L$ is the optical phase accumulated in this distance, with $\beta=\left(2 \pi \cdot n_{\text {eff }}\right) / \lambda$. Using the condition relating the coupler output to the input $a_{1}=\alpha \cdot e^{i \beta L} \cdot b_{1}$ yields a solution of the matrix equation:

$$
\left|\frac{b_{0}}{a_{0}}\right|^{2}=\frac{\alpha^{2}+|t|^{2}-2 \alpha|t| \cos (\beta \cdot L)}{1+\alpha^{2}|t|^{2}-2 \alpha|t| \cos (\beta \cdot L)}
$$

which on resonance $(\beta \cdot L=2 \pi \cdot m$ for some integer $m)$ is

$$
\left|\frac{b_{0}}{a_{0}}\right|^{2}=\frac{(\alpha-|t|)^{2}}{(1-\alpha|t|)^{2}}
$$

The condition $\alpha=|t|$ is referred to as critical coupling [3]. Thus, when the waveguide is critically coupled to the ring resonator, the filter response will be a series of nulls periodic in wavelength.

Results and discussion: As with most polymeric optical devices, we begin by spinning the lower cladding and core layers on a silicon substrate. In our case, the lower cladding, core and upper cladding materials are commercially available polymers UV-15 (Masterbond), SU-8 (Microchem) and OG-125 (Epotek), with refractive indices $1.50,1.57$ and 1.46 , respectively, at $1550 \mathrm{~nm}$. The crucial interface for the realisation of the free-standing devices is that between the lower cladding and the substrate. We have observed that UV-15, spun to a thickness of 5-10 $\mu \mathrm{m}$ and cured under an ultraviolet (UV) lamp, displays only weak adhesion to a layer of gold evaporated on silicon. The $2 \mu \mathrm{m}$-wide and $1.6 \mu \mathrm{m}$-thick waveguide cores are formed by directly cross-linking the SU-8 using electron beam lithography and subsequent developing in SU-8 developer. A $2.5 \mu \mathrm{m}$ upper cladding is cured under the UV lamp. Using the weak adhesion of the interface between the lower cladding and the gold, multiple devices are easily peeled up as a flexible film with dimensions of several square centimetres and approximately $10 \mu \mathrm{m}$ thick. Given a free-standing film containing many optical devices, preparation of the waveguide end-facets for device measurement presents a difficulty, as there is no substrate to dice and polish. We find that good optical quality endfacets can be produced by simply cutting the film using a surgical scalpel blade, a procedure to be described elsewhere.
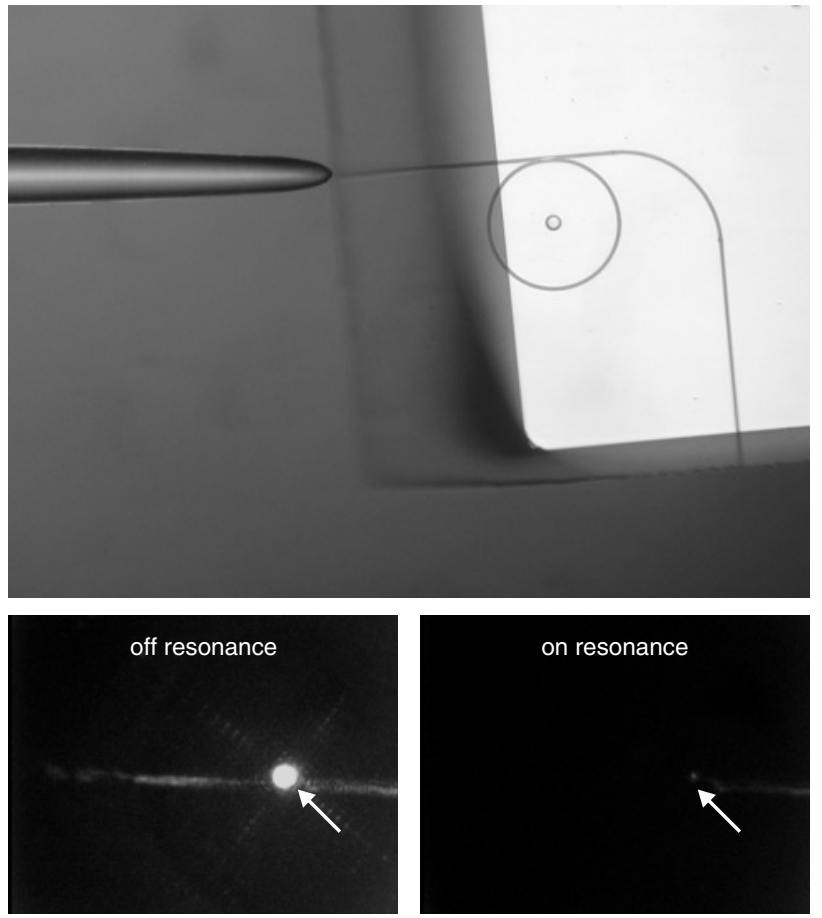

Fig. 2 Free-standing all-polymer microring resonator filter (semitransparent) placed upon, but not adhered to, silicon wafer (white in photograph) for support purposes only during optical measurement. Tapered input fibre shown on left. CCD images of output, off-resonance (bottom left) and on-resonance (bottom right)

A free-standing filter device fabricated in this manner, as shown in Fig. 2, is placed, but not adhered to, a silicon wafer for support purposes only during measurement. Single polarisation light from a tunable diode laser is input into the device using a tapered singlemode fibre with a focused spot size of approximately $1.5 \times 3 \mu \mathrm{m}$. A $20 \times$ microscope objective focuses the output signal onto a $\mathrm{CCD}$, as in Fig. 2, or a photodetector for measurement. For spectral interrogation of the devices, both the input wavelength scans and the photodetector measurement are controlled by computer. Fig. 3 shows the output 
spectrum (circles) for the free-standing microring optical filter as measured over an input wavelength scan of $10 \mathrm{~nm}$. As previously mentioned, the output should consist of a periodic series of nulls in wavelength, given by (2). The theoretical fit of the data is shown in Fig. 3 (solid line) demonstrating good agreement between the measured device and the theoretical expectations. The output nulls occur periodically with spacing equal to the free-spectral range FSR $=c /\left(n_{\text {eff }} \cdot L\right)$ of approximately $2.4 \mathrm{~nm}(300 \mathrm{GHz})$. The extinction depths of the nulls are measured to be $-27 \mathrm{~dB}$ of the maximum output signal, which is the best value yet reported for similar polymeric devices, whether free-standing or not. However, this extinction value is the minimum signal limit of the measurement apparatus due to the background noise, and the theoretical fit predicts a slightly lower value. The level of filtering demonstrated here is within the range demanded of commercial components.

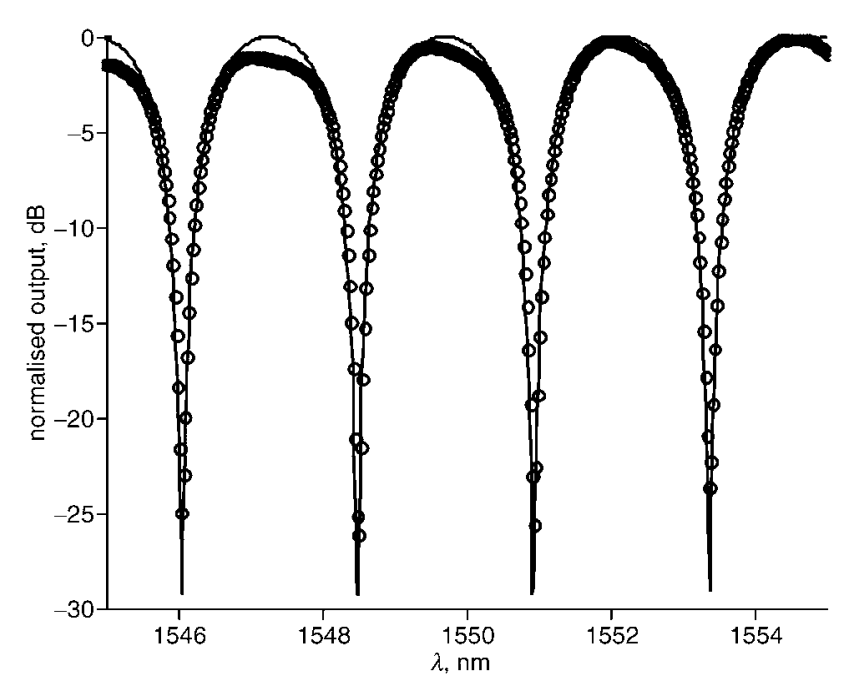

Fig. 3 Measured spectral response of optical filter and theoretical fit $\bigcirc$ measured $\quad-$ theoretical
Conclusion: We have presented a free-standing all-polymer microring resonator with optical filter characteristics which exceed those previously reported in polymeric materials, whether free-standing or not. Such a result serves as a demonstration to the potential of flexible polymer integrated optical circuits in optical communications. An intriguing use of these free-standing polymer optical devices is the ability to conform to various surfaces and materials with specific properties. For example, one of the most severe limitations preventing deployment of polymer optical devices is the refractive index change of the material due to temperature fluctuations. Flexible all-polymer devices can be adhered to active cooling substrates, thus offsetting deleterious thermo-optic deviations of desired device performance.

(C) IEE 2003

Electronics Letters Online No: 20031090

28 July 2003 DOI: 10.1049/el:20031090

G.T. Paloczi, Y. Huang and A. Yariv (Department of Applied Physics, California Institute of Technology, Pasadena, CA 91125, USA)

\section{References}

1 SHI, Y., et al.: 'Low (sub-1-volt) halfwave voltage polymeric electro-optic modulators achieved by controlling chromophore shape', Science, 2000, 288, pp. 119-122

2 LEE, M., et al.: 'Broadband modulation of light by using an electro-optic polymer', Science, 2002, 298, pp. 1401-1403

3 YARIV, A.: 'Universal relations for coupling of optical power between microresonators and dielectric waveguides', Electron. Lett., 2000, 36, (4), pp. $321-322$ 